\title{
Functional Training Protocol in Men with Prostate Cancer: A Non-Randomized Clinical Trial
}

\section{Taysi Seemann $^{1^{*}}$, Anke Bergmann ${ }^{2}$, Leonessa Boing ${ }^{1}$, Magnus Benetti ${ }^{1}$, Melissa de Carvalho Souza Vieira $^{1}$ and Adriana Coutinho de Azevedo Guimaraes ${ }^{1}$}

${ }^{1}$ Department of Physical Education, Health and Sports Science Center, State University of Santa Catarina, Brazil

${ }^{2}$ Clinical Epidemiology Program and Molecular Carcinogenesis Program, National Cancer Institute (INCA), Brazil

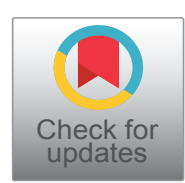

*Corresponding author: Taysi Seemann, Master Student, Department of Physical Education, Health and Sports Science Center, State University of Santa Catarina, General Estilac Leal, 129-101C, Florianópolis, Santa Catarina, Brazil, Tel: +5548999591421,E-mail: taysiseemann@gmail.com

\begin{abstract}
Prostate cancer treatment is associated with side effects including urinary incontinence, erectile dysfunction, fatigue, depressive symptoms, and a decrease in physical function. Physical exercise has been considered beneficial in countering these side effects. We believe that supervised exercise programs may be an efficient alternative to treatment and rehabilitation in these patients. As part of an ongoing analysis, we sought to evaluate a 12-week physical exercise protocol in prostate cancer survivors. Patients will be divided into two groups (intervention and control) and data were collected before and after the study. We hope to identify whether physical exercise is effective in prostate cancer survivors, and establish recommendations for specific exercises, nowadays this measures are so restricted especially.
\end{abstract}

\section{Keywords}

Prostate neoplasm, Physical exercise, Exercise program, Protocol, Cancer

\section{Introduction}

Prostate cancer has become a health public concern [1]. It is the sixth most common cancer in the world, the most prevalent cancer among men, and represents approximately $10 \%$ of all cancers [2]. In 2016, it was estimated that 295,200 men in Brazil had cancer, including 61,200 with prostate cancer [1]. Owing to advances in the detection and medical treatment of this condition, patients' survival rate has steadily increased [3]. However, a large proportion of prostate cancer survivors have side effects associated with the disease and its treatment, including urinary incontinence, erectile dysfunction, fatigue, depressive symptoms, anguish, and a decrease in physical function [4-6]. Fortunately, studies have demonstrated that regular physical exercise can minimize these side effects [7].

Current literature emphasizes the importance of regular physical activity in the general population $[8,9]$. Physical activity has also been shown to be beneficial in patients with cancer who have psychological and physical concerns [7]. At least 150 minutes of moderate or 75 minutes of intensive physical activity per week is recommended in patients with cancer [8]. The American College of Sports Medicine (ACSM) has similar recommendations and further suggests that resistance training be performed twice a week to improve general physical health [10]. In addition, researchers have suggested a combination of resistance and aerobic training in men with prostate cancer [11], because these patients tend to have treatment-related muscle loss, cardiorespiratory decline, urinary incontinence, and muscular fatigue.

However, data on physical activity protocols in patients with cancer are lacking. The only physical activities that have been evaluated include walking [12] and aerobic exercise combined with resistance training [1315]. In addition, some studies have assessed behavioral support [16-18] and pelvic floor training [19]. Functional training is one of the modalities that combine resis- 
tance training, aerobic exercise, and pelvic floor training to improve people's functional capacity, daily activities, autonomy, and independence [20].

It is possible that this kind of activity is the most effective intervention in patients with prostate cancer. Previous researchers have reported deterioration of physical fitness associated with cancer and its treatment [2], decreasing muscle mass and strength, increasing weight and body fat, and decreasing in the physical functions [3]. Changes that could suffer improvement with regular practice of functional training [20], because it is based on variables of physical fitness provide a global evolution of the individual. In addition, physical exercises should include pelvic floor training to treat the urinary incontinence, which is associated with radical prostatectomy surgery, the oldest and possible more efficient measure to treat local prostate cancer [21].

In this study, we will be conducting a non-randomized clinical trial to evaluate the effectiveness a 12-week functional training protocol consisting of twice weekly exercise in an Experimental Group (EG) of patients with prostate cancer, and compared it to a Control Group (CG) of patients who did not perform any physical exercise. We hypothesize that patients in the EG will have improved quality-of-life, sexual activity, and physical fitness, as well as less fatigue and depression-related symptoms, compared with the CG.

\section{Methods}

\section{Research type}

Non-randomized clinical trial with a EG undergoing a 12-week intervention period and a CG.

\section{Participants of study}

Patients diagnosed with prostate cancer currently undergoing treatment or who have completed treatment will be included in the non-probabilistic sample.

Patients between $50-80$ years will be included in the study. This age range was selected because it has the highest prevalence of prostate cancer, and patients are undergoing adjuvant or neoadjuvant treatment, or have completed treatment. Criteria for exclusion will include patients who are illiterate and those whose disease metastasized. The latter criterion was selected because of the variability of local metastases as it relates to treatment and prognosis. Furthermore, patients who exercised three months before the beginning of the study will be excluded from the analysis.

We estimated that 38 patients will be needed in the study based on sample size calculations using $G *$ Power 3.1.9.2, with effect size of 0.67 , level of significance of $5 \%$, power of the test of $90 \%$, and sample loss of $20 \%$ [22]. Patients will be divided into two groups: The CG ( $=19)$ and the EG $(n=19)$. Data will be collected before, during, and after the 12-week study period; patients will also attend lectures twice a week. Patients in the EG will undergo a 12-week functional training intervention, and be invited to continue the protocol after the conclusion of the study.

\section{Data collection}

The intervention group: The protocol will be executed in a fitness center that focuses on functional training located in the mainland area of Florianopolis. We will use the local press to raise awareness about the study and recruit patients. Patients will also be recruited from the "Centro de Pesquisas Oncologicas" (CEPON) Oncological Research Center, in Florianopolis. Patients will be contacted by phone and invited to participate in the study. Procedures including prior collection of data, application of the functional training, and post-study data collection will be explained; the importance of the assiduity will also be discussed. Once patients agree to follow the study protocol, they will be asked to sign a consent form, after which point data collection will begin. The initial assessment will consist of a quiz (Table 1 ) and physical fitness test (Table 2). Both will occur one week before the intervention at a silent location to ensure that patients understand the quiz. The intervention will be initiated after the initial data collection, and last one hour twice weekly in accordance with the study protocol. The same quiz and physical fitness test will be administered at the conclusion of the 12-week intervention.

The protocol will include 24 different types of training. Functional exercise will comprise agility, balance, flexibility, resistance, potency, coordination, and strength. Each week, agility will be combined with another physical fitness variable. Each session will last one hour. Each session will consist of a 10-minute warm-up, followed by a 10-minute warm-up specific to an exercise, 30-minute of exercise, and a 10-minute cool down [7]. The first week of the intervention will focus on pelvic strength training, which will help improve the overall performance of the patients and urinary incontinence urinary. Aerobic activity will be performed through agility exercises during the warm-up session, whereas the main 30-minute exercise session will consist of resistance training. The warm-up and main exercise will be performed in cycles of 5 different types of exercises, where patients perform 8-12 repetitions for each circuit, 1 to 3 times, while respecting the physical limits of each patient.

The control group: Data in the CG will be simultaneously collected with the EG. The personal involved will be included in case of a lack of interest or possibility in being a part of the EG. We will schedule an at-home patient visit or meet with them at another location of their choosing. We will then thoroughly explain the purpose of the study, highlighting the importance of regular physical activity, and request that the patient does not initiate any physical activity similar to the intervention. To keep patients in the CG involved, they will attend bi- 
Table 1: Functional training protocol for men diagnosed with prostate cancer.

\begin{tabular}{|c|c|c|c|c|}
\hline & Training & Warm-Up & Main Exercise & Cool Down \\
\hline \multirow{2}{*}{ 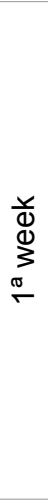 } & & $\begin{array}{l}\text { Agility } \\
\text { 1- Single-leg pendulum } \\
\text { 2- One-leg dumbbell squats } \\
\text { 3- Knee hug } \\
\text { 4- Rope jumping } \\
\text { 5- Pendulum balancing disk } \\
\left(\mathrm{PF}^{*}\right)\end{array}$ & $\begin{array}{l}\text { Pelvis Recruitment } \\
\text { 1- Teaching the posture to be } \\
\text { used during the training; } \\
\text { 2- Breathing for training: } \\
\text { 3- Correct pelvis recruitment }\end{array}$ & $\begin{array}{l}\text { Stretching rope } \\
\text { exercises }\end{array}$ \\
\hline & & $\begin{array}{l}\text { Agility } \\
\text { 1- Lateral jumping jacks } \\
\text { 2- Free squats } \\
\text { 3- Turn exercise } \mathrm{BS}^{*} \\
\text { 4- Rotating arms } \\
\text { 5- Leg circles }\left(\mathrm{PF}^{*}\right)\end{array}$ & $\begin{array}{l}\text { Pelvis Recruitment } \\
\text { 1- Teaching the posture to be } \\
\text { used during the training; } \\
\text { 2- Breathing for training: } \\
\text { 3- Correct pelvis recruitment }\end{array}$ & Stretching exercises \\
\hline \multirow{2}{*}{ 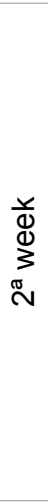 } & & $\begin{array}{l}\text { Agility } \\
\text { 1- Jogging } \\
\text { 2- Trunk twists } \\
\text { 3- Hacky sack exercise } \\
\text { 4- Pendulum lying exercise } \\
\text { 5- Calf squats }\left(\mathrm{PF}^{*}\right)\end{array}$ & $\begin{array}{l}\text { Flexibility } \\
\text { 1- Sumo squat }\left(\mathrm{PF}^{*}\right) \\
\text { 2- Side ABD* abduction } \\
\text { 3- Last side } \\
\text { 4- Switchblade } \\
\text { 5- Free squats }\end{array}$ & $\begin{array}{l}\text { Stretching rope } \\
\text { exercises }\end{array}$ \\
\hline & & $\begin{array}{l}\text { Agility } \\
\text { 1- Agility ladder } \\
\text { 2- Front jumping jacks } \\
\text { 3- Calf exercise } \\
\text { 4- Compound squats }\left(\mathrm{PF}^{*}\right) \\
\text { 5- Alternating arms }\end{array}$ & $\begin{array}{l}\text { Flexibility } \\
\text { 1- Free squatss } \\
\text { 2- Frog squats } \\
\text { 3- Calf squats (PF*) } \\
\text { 4- Sink while stretching } \\
\text { 5- "Y" and "T" in suspension tape }\end{array}$ & Stretching exercises \\
\hline \multirow{2}{*}{ 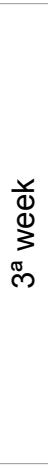 } & & $\begin{array}{l}\text { Agility } \\
\text { 1- Jumping squat on a step } \\
\text { 2- Lateral jumping jacks } \\
\text { 3- Calf squats }\left(\mathrm{PF}^{\star}\right) \\
\text { 4- Single-leg pendulum } \\
\text { 5- Waist turning } \mathrm{BS}^{*}\end{array}$ & $\begin{array}{l}\text { Endurance exercise } \\
\text { 1- Strength } \\
\text { 2- Remadasuspension tape } \\
\text { 3- Push-ups }\left(\mathrm{PF}^{*}\right) \\
\text { 4- Side elevation } \\
\text { 5- } \mathrm{ABD}^{*} \text { supra } \mathrm{MB}^{*}\end{array}$ & $\begin{array}{l}\text { Stretching rope } \\
\text { exercises }\end{array}$ \\
\hline & & $\begin{array}{l}\text { Agility } \\
\text { 1- } C^{*} \text { Barriers } \\
\text { 2- } C^{*} \text { High knee skips } \\
\text { 3- } C^{*} \text { Cross crawl } \\
\text { 4- } C^{*} \text { Front raises } \\
\text { 5- } C^{*} \text { Side raises }\end{array}$ & $\begin{array}{l}\text { Endurance exercise } \\
\text { 1- Supine } \mathrm{BS}^{*} \\
\text { 2- Bridge }\left(\mathrm{PF}^{*}\right) \\
\text { 3- Strength } \\
\text { 4- Last side } \\
\text { 5- Free squats }\left(\mathrm{PF}^{*}\right)\end{array}$ & $\begin{array}{l}\text { Stretching rope } \\
\text { exercises }\end{array}$ \\
\hline \multirow{2}{*}{ 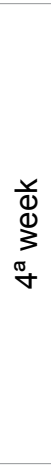 } & & $\begin{array}{l}\text { Agility } \\
\text { 1- Up on the tire } \\
\text { 2- Marine rope } \\
\text { 3- Launch medicine ball exercise } \\
\text { 4- Jump cones } \\
\text { 5- Leg circles }\left(\mathrm{PF}^{*}\right)\end{array}$ & $\begin{array}{l}\text { Power exercises } \\
\text { 1- Bridge }\left(\mathrm{PF}^{*}\right) \\
\text { 2- } \mathrm{ABD}^{*} \mathrm{MB}^{*} \text { in wall } \\
\text { 3- Punch } \\
\text { 4- } \mathrm{C}^{*} \text { high knee skips } \\
\text { 5- Tríceps }\end{array}$ & Lying down stretching \\
\hline & & $\begin{array}{l}\text { Agility } \\
\text { 1- Trampoline } \\
\text { 2- Hacky sack exercise } \\
\text { 3- Agility ladder } \\
\text { 4- Calf squats }\left(\mathrm{PF}^{\star}\right) \\
\text { 5- Calf raises }\end{array}$ & $\begin{array}{l}\text { Power exercises } \\
\text { 1- Free squats with } \mathrm{MB}^{*} \\
\text { 2- Sink + front and side elevation } \\
\text { 3- Pitch } \mathrm{MB}^{*} \\
\text { 4- Calf squat } \\
\text { 5- } \mathrm{ABD}^{*} \text { rower }\end{array}$ & Lying down stretching \\
\hline \multirow[b]{2}{*}{ 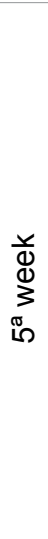 } & & $\begin{array}{l}\text { Agility } \\
\text { 1- Hug knee forward/backward }\left(\mathrm{PF}^{\star}\right) \\
\text { 2- Calf with hands up } \\
\text { 3- Power skips exercise } \\
\text { 4- Low impact exercise } \\
\text { 5- Scorpion stretch }\end{array}$ & $\begin{array}{l}\text { Coordination } \\
\text { 1- } C^{*} \text { Barriers } \\
\text { 2- Support step + jump } \\
\text { 3- Board } \\
\text { 4- } \mathrm{ABD}^{*} \text { rower }\left(\mathrm{PF}^{\star}\right) \\
\text { 5- Supine UNI* }\end{array}$ & $\begin{array}{l}\text { Stretching rope } \\
\text { exercises }\end{array}$ \\
\hline & 10 & $\begin{array}{l}\text { Agility } \\
\text { 1- Pendulum Balancing disk }\left(\mathrm{PF}^{\star}\right) \\
\text { 2- Gym ball passing jackknife } \mathrm{MB}^{*} \\
\text { 3- Rope jumping } \\
\text { 4- Jump on tire } \\
\text { 5- One-leg dumbbell squat } \\
\left(\mathrm{PF}^{\star}\right)\end{array}$ & $\begin{array}{l}\text { Coordination } \\
\text { 1- Power skip exercise } \\
\text { 2- Swing }\left(\mathrm{PF}^{*}\right) \\
\text { 3- ABD supra } \\
\text { 4- Squats + remadasuspension } \\
\text { tape } \\
\text { 5- STEP }\end{array}$ & $\begin{array}{l}\text { Myofascial release } \\
\text { exercises }\end{array}$ \\
\hline
\end{tabular}




\begin{tabular}{|c|c|c|c|}
\hline \multirow{2}{*}{ 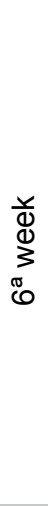 } & $\begin{array}{l}\text { 11. Agility } \\
1-C^{*} \text { high knee drills } \\
2-C^{*} \text { rotation exercise } \\
3-C^{*} \text { plantar fasciitis } \\
4-C^{*} \text { side } \\
5-C^{*} \text { back }\end{array}$ & $\begin{array}{l}\text { Strength exercises } \\
\text { 1- Free squats }\left(\mathrm{PF}^{*}\right) \\
\text { 2- Board } \\
\text { 3- Barbell } \\
\text { 4- Supine } \\
\text { 5- Squats sumô }\left(\mathrm{PF}^{*}\right)\end{array}$ & Stretching exercises \\
\hline & $\begin{array}{l}\text { 12. Agility } \\
\text { 1- } \mathrm{C}^{*} \text { Barriers } \\
\text { 2- Hacky sack exercise } \\
\text { 3- Agility ladder } \\
\text { 4- Front jumping jacks } \\
\text { 5- Turn exercise } \mathrm{MB}^{*}\left(\mathrm{PF}^{*}\right)\end{array}$ & $\begin{array}{l}\text { Strength exercises } \\
\text { 1- Stiff }\left(\mathrm{PF}^{*}\right) \\
\text { 2- Bridge UNI* }\left(\mathrm{PF}^{*}\right) \\
\text { 3- Front elevation } \\
\text { 4- Squat in balancing disk }\left(\mathrm{PF}^{*}\right) \\
\text { 5- } \mathrm{ABD}^{*} \text { infra }\end{array}$ & $\begin{array}{l}\text { Myofascial release } \\
\text { exercises }\end{array}$ \\
\hline \multirow{2}{*}{ 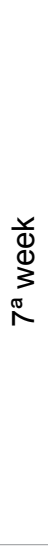 } & $\begin{array}{l}\text { 13. Agility } \\
\text { 1- Escalator } \\
\text { 2- Gym ball passing jackknife MB* } \\
\text { 3- Rope jumping } \\
\text { 4- Jumping on tire } \\
\text { 5- Plane exercise }\end{array}$ & $\begin{array}{l}\text { Balance } \\
\text { 1- Walking on rope } \\
\text { 2- Barbell on the ball } \\
\text { 3- Squats + rowing with } \\
\text { elasticized } \\
\text { 4- Plane }\left(\mathrm{PF}^{*}\right) \\
\text { 5- Stiff UNI }\left(\mathrm{PF}^{*}\right)\end{array}$ & Lying down stretching \\
\hline & $\begin{array}{l}\text { 14. Agility } \\
\text { 1- } \mathrm{C}^{*} \text { barrier } \\
\text { 2- Scorpion stretch } \\
\text { 3- Scorpion back stretch } \\
\text { 4- Hip bridge exercise }\left(\mathrm{PF}^{*}\right) \\
\text { 5- Leg circles }\left(\mathrm{PF}^{*}\right)\end{array}$ & $\begin{array}{l}\text { Balance } \\
\text { 1- Squat } \mathrm{MB}^{*}\left(\mathrm{PF}^{*}\right) \\
\text { 2- Side } \mathrm{ABD}^{*} \mathrm{SB}^{*} \\
\text { 3- Supine } \mathrm{SB}^{*} \\
\text { 4- Pendulum bosu row }\left(\mathrm{PF}^{*}\right) \\
\text { 5- Perdigueiro }\end{array}$ & Lying down stretching \\
\hline \multirow{2}{*}{$\begin{array}{l}\text { ऐ. } \\
\underset{\infty}{\infty} \\
\infty \\
\infty\end{array}$} & $\begin{array}{l}\text { 15. Agility } \\
\text { 1- Agility ladder } \\
\text { 2- Hacky sack exercise } \\
\text { 3- Jumping on tire } \\
\text { 4- Rope jumping } \\
\text { 5- Calf raises }\end{array}$ & $\begin{array}{l}\text { Flexibility } \\
\text { 1- Scorpion } \\
\text { 2- Stretching exercise } \\
\text { 3- } \mathrm{ABD}^{*} \mathrm{SB}\left(\mathrm{PF}^{*}\right) \\
\text { 4- Parachute } \\
\text { 5- Standing side stretch } \mathrm{SB}^{*}\end{array}$ & Stretching exercises \\
\hline & $\begin{array}{l}\text { 16. Agility } \\
\text { 1- } \mathrm{C}^{*} \text { barrier } \\
\text { 2- Scorpion stretch } \\
\text { 3- Scorpion back stretch } \\
\text { 4- Hip bridge exercise }\left(\mathrm{PF}^{*}\right) \\
\text { 5- Leg circles }\left(\mathrm{PF}^{*}\right)\end{array}$ & $\begin{array}{l}\text { Flexibility } \\
\text { 1- Frog squats } \\
\text { 2- Sumo squat }\left(\mathrm{PF}^{\star}\right) \\
\text { 3- Last side } \\
\text { 4- Switchblade } \\
\text { 5- Free squats }\end{array}$ & Lying down stretching \\
\hline \multirow{2}{*}{ 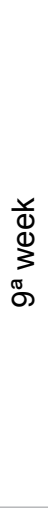 } & $\begin{array}{l}\text { 17. Agility } \\
\text { 1- Jumping rope } \\
\text { 2- Clap jacks forward/backward } \\
\text { 3- Front jumping jacks } \\
\text { 4- Hip extension and flexion }\left(\mathrm{PF}^{\star}\right) \\
\text { 5- Calf raises }\end{array}$ & $\begin{array}{l}\text { Endurance exercise } \\
\text { 1- Marine rope } \\
\text { 2- } \mathrm{ABD}^{*} \text { bicicleta }\left(\mathrm{PF}^{*}\right) \\
\text { 3- Remada in suspension tape } \\
\text { 4- Bridge }\left(\mathrm{PF}^{*}\right) \\
\text { 5- Supine }\end{array}$ & Stretching exercises \\
\hline & $\begin{array}{l}\text { 18. Agility } \\
\text { 1- } \mathrm{C}^{*} \text { Barriers } \\
\text { 2- Jumping on tire } \\
\text { 3- Leg circles UNI* }\left(\mathrm{PF}^{*}\right) \\
\text { 4- Seal jacks } \\
\text { 5- Jumping on tire }\end{array}$ & $\begin{array}{l}\text { Endurance exercise } \\
\text { 1- Supine } \mathrm{BS}^{*} \\
\text { 2- Bridge }\left(\mathrm{PF}^{*}\right) \\
\text { 3- Strength } \\
\text { 4- Last side } \\
\text { 5- Free squats }\left(\mathrm{PF}^{*}\right)\end{array}$ & Lying down stretching \\
\hline \multirow{2}{*}{ 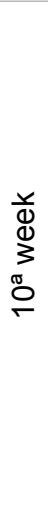 } & $\begin{array}{l}\text { 19. Agility } \\
\text { 1- } \mathrm{C}^{*} \text { Barriers } \\
\text { 2- Scorpion stretch } \\
\text { 3- Hip bridge exercise }\left(\mathrm{PF}^{*}\right) \\
\text { 4- Lateral jumping jacks } \\
\text { 5- Turn exercise } \mathrm{MB}^{*}\end{array}$ & $\begin{array}{l}\text { Power exercises } \\
\text { 1- Jumping on step } \\
\text { 2- Free squats with } \mathrm{MB}^{*} \\
\text { 3- } \mathrm{ABD}^{*} \text { rower } \\
\text { 4- Pitch } \mathrm{MB}^{*} \\
\text { 5- Calf squat }\end{array}$ & Stretching exercises \\
\hline & $\begin{array}{l}\text { 20. Agility } \\
\text { 1- Front jumping jacks } \\
\text { 2- Hand to foot jack exercise } \\
\text { 3- Lateral jumping jacks } \\
\text { 4- Scorpion back stretch } \\
\text { 5- Hip bridge exercise }\left(\mathrm{PF}^{\star}\right)\end{array}$ & $\begin{array}{l}\text { Power exercises } \\
\text { 1- Bridge }\left(\mathrm{PF}^{*}\right) \\
\text { 2- Push-up } \\
\text { 3- Punch } \\
\text { 4- } C^{*} \text { High knee skips } \\
\text { 5- Triceps }\end{array}$ & $\begin{array}{l}\text { Myofascial release } \\
\text { exercises }\end{array}$ \\
\hline
\end{tabular}




\begin{tabular}{|c|c|c|c|}
\hline \multirow{2}{*}{$\begin{array}{l}\text { y } \\
\Phi \\
\vdots \\
\vdots \\
\sigma \\
\sigma\end{array}$} & $\begin{array}{l}\text { 21. Agility } \\
\text { 1- } C^{*} \text { Barriers } \\
\text { 2- } C^{*} \text { high knee skips } \\
\text { 3- } C^{*} \text { cross crawl } \\
4-C^{*} \text { front raises } \\
5-C^{*} \text { side raises }\end{array}$ & $\begin{array}{l}\text { Coordination } \\
\text { 1- } \mathrm{C}^{*} \text { barriers } \\
\text { 2- Support step + jump } \\
\text { 3- Agility ladder } \\
\text { 4- ABD rower }\left(\mathrm{PF}^{*}\right) \\
\text { 5- Supine UNI* }\end{array}$ & Stretching exercises \\
\hline & $\begin{array}{l}\text { 22. Agility } \\
\text { 1- Trunk rotation exercises } \\
\text { 2- } \mathrm{C}^{*} \text { cross crawl } \\
\text { 3- Scorpion stretch } \\
\text { 4- Front jumping jacks } \\
\text { 5- Rower exercise }\left(\mathrm{PF}^{*}\right)\end{array}$ & $\begin{array}{l}\text { Coordination } \\
\text { 1- Power skips exercise } \\
\text { 2- Swing }\left(\mathrm{PF}^{*}\right) \\
\text { 3- STEP } \\
\text { 4- Squats + remada } \\
\text { 5- STEP }\end{array}$ & $\begin{array}{l}\text { Myofascial release } \\
\text { exercises }\end{array}$ \\
\hline \multirow{2}{*}{ 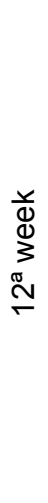 } & $\begin{array}{l}\text { 23. Agility } \\
\text { 1- Single leg pendulum } \\
\text { 2- One leg dumbbell squat } \\
\text { 3- Knee hug } \\
\text { 4- Rope jumping } \\
\text { 5- Pendulum balancing disk }\left(\mathrm{PF}^{*}\right)\end{array}$ & $\begin{array}{l}\text { Strength exercises- } \\
\text { 1- Free squats }\left(\mathrm{PF}^{*}\right) \\
\text { 2- Board } \\
\text { 3- Barbell } \\
\text { 4- Supine } \\
\text { 5- Squats sumô }\left(\mathrm{PF}^{*}\right)\end{array}$ & Stretching exercises \\
\hline & $\begin{array}{l}\text { 24. Agility } \\
1-C^{*} \text { barriers } \\
2-C^{*} \text { high knee skips } \\
3-C^{*} \text { cross crawl } \\
4-C^{*} \text { front raises } \\
5-C^{*} \text { side raises }\end{array}$ & $\begin{array}{l}\text { Strength exercises } \\
\text { 1- Stiff }\left(\mathrm{PF}^{\star}\right) \\
\text { 2- Bridge UNI }\left(\mathrm{PF}^{\star}\right) \\
\text { 3- Front elevation } \\
\text { 4- Squat bozú }\left(\mathrm{PF}^{\star}\right) \\
\text { 5- } \mathrm{ABD}^{*} \text { infra }\end{array}$ & $\begin{array}{l}\text { Myofascial release } \\
\text { exercises }\end{array}$ \\
\hline
\end{tabular}

"SB: Swiss Ball; ABD: Abdominal; UNI: Unilateral; MB: Medicine Ball; PF: Pelvic Floor; C: run.

Table 2: Physical test.

\begin{tabular}{|l|l|l|}
\hline Variable & Test & Source \\
\hline Flexibility & "Sit and reach" & {$[25]$} \\
\hline Resistence & Abdominal in one minute & {$[26]$} \\
\hline Power exercises & Push up & {$[26]$} \\
\hline Balance and agility & $\begin{array}{l}\text { Agility and dynamic balance } \\
\text { test: Protocol AAHPERD }\end{array}$ & {$[27]$} \\
\hline Coordination & $\begin{array}{l}\text { Coordination test: Protocol } \\
\text { AAHPERD }\end{array}$ & {$[27]$} \\
\hline Power rating & Throw the medicine ball & {$[28]$} \\
\hline
\end{tabular}

weekly lectures on topics including health maintenance, lesion prevention, and nutrition. Post-study data will be collected in patients who did perform physical activity during the study, but their data will be excluded from the analysis. The picture 01 shows the participants selection process and the execution of the study protocol (Figure 1).

We will conduct biweekly meetings with the assistance of other professionals in the field of nutrition and physiotherapy. These meetings will be scheduled in advance and conducted at the same location as the intervention. They will last 30 minutes and consist of motivational lectures and basic practical guidelines for physical activity, including appropriated clothing and hydration.

A total of six meetings will be schedule; the topic of each meeting is listed in (Table 3).

\section{Measures of results}

Although validated protocols are lacking in this patient population most of the resistance training and aerobic exercises used in the intervention will already be familiar to the patients in our study and have been eval-
Table 3: Meetings control group.

\begin{tabular}{|l|l|}
\hline Meeting & Subject \\
\hline 1 & Introduction to the project and address concerns \\
\hline 2 & Benefits of practical of physical activity \\
\hline 3 & Motivation to do a physical activity \\
\hline 4 & Physical activity and diseases prevention \\
\hline 5 & Nutrition for the practical of physical activity \\
\hline 6 & Physical lesions prevention \\
\hline
\end{tabular}

uated in previous studies $[13-15,23]$. We expect that the functional training intervention will provide positive physical and psychological results in this patient population (Table 4).

\section{Statistical analysis}

An electronic spreadsheet will be made in Excel XP and data moved to the statistic package SPSS (IBM version 20.0). Descriptive statistics including average, standard curve (divert, diversion) and percentages will be calculated first so that we can understand the characteristics of the patient population. The Kolmogorov-Smirnov test will be used to determine the normal distribution of the outcomes; the normal will be set to $p<0.05$. The average of the different outcomes will be calculated and compared between groups. Statistical differences will be obtained using variance analysis.

Univariate linear regression will be used to determine the $\beta$ coefficient of each outcome and adjust for potential confounders in each group. Variables with $p<0.20$ will be selected to produce a multi-linear regression model; $p$ values $<0.05$ will be considered statistically significant. The homoscedasticity will be analyzed and possible bias of the model through residual analyses, and all assumptions 


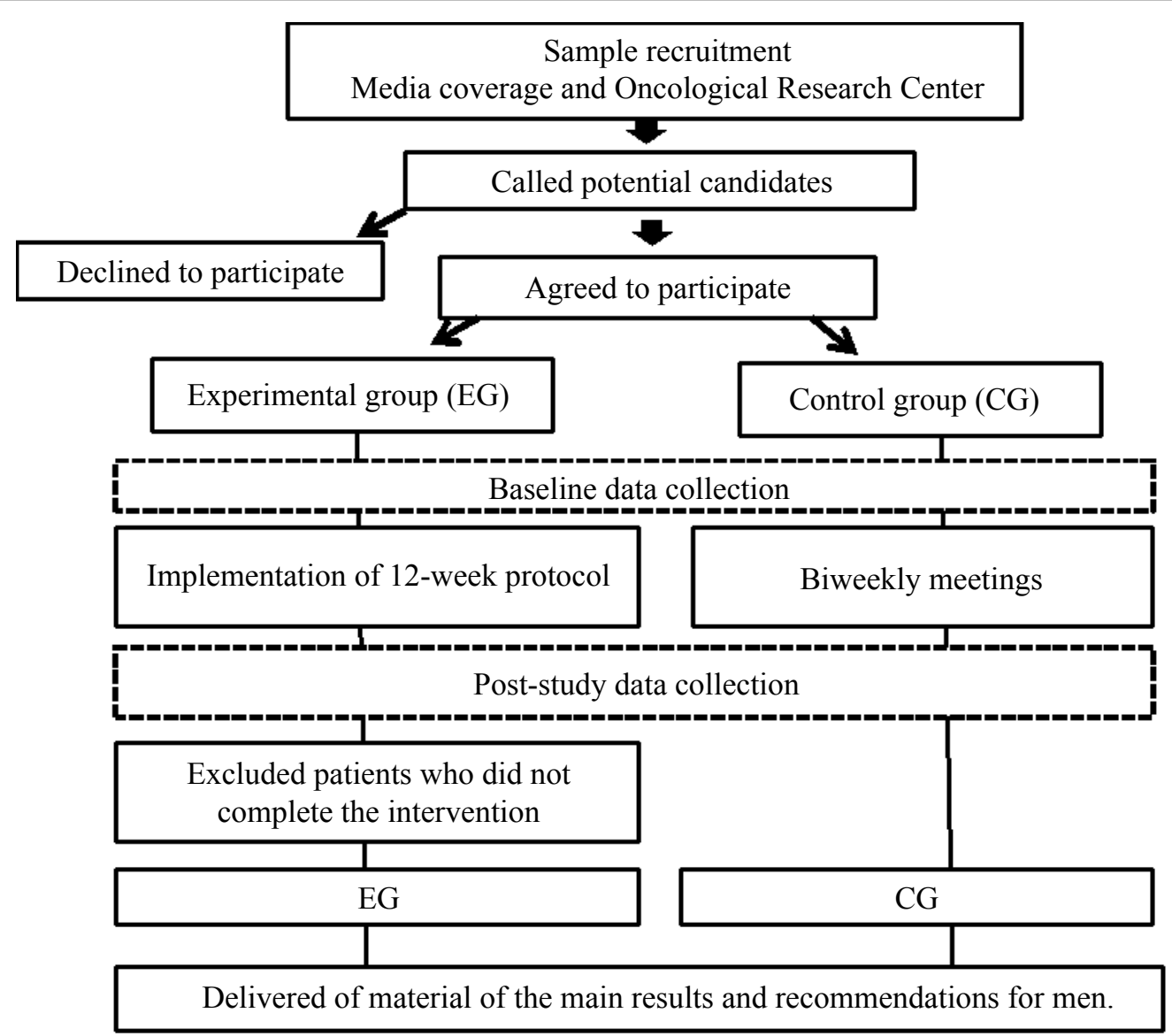

Figure 1: Flow chart of the selection process.

Table 4: Measures of results.

\begin{tabular}{|c|c|c|c|}
\hline \multirow{3}{*}{ 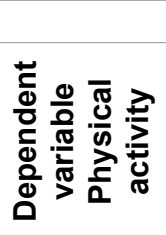 } & Variables & Definitions & Criteria \\
\hline & \multicolumn{3}{|l|}{ Functional training } \\
\hline & Level of physical activity & $\begin{array}{l}\text { Insufficiently active, active, } \\
\text { sufficiently active }\end{array}$ & $\begin{array}{l}\text { IPAQ (short version) international quiz of physical } \\
\text { activity [29]. }\end{array}$ \\
\hline \multirow{6}{*}{ 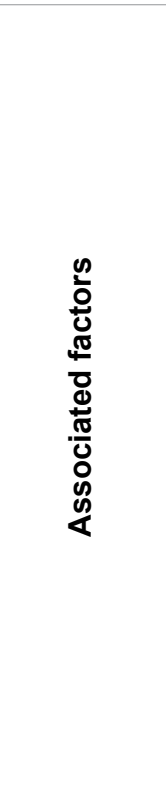 } & Quality-of-life & $\begin{array}{l}\text { The higher the score, the better } \\
\text { quality-of-life } \\
\text { Scores range from } 0-100 \\
\text { Functional scale and global health: } \\
\text { The closer of } 100 \text {, the better the } L Q \text {; } \\
\text { Symptomatic scale: The closer to } \\
100 \text {, the better the LQ. }\end{array}$ & $\begin{array}{l}\text { EORTC QLQ-C30 European Organization for } \\
\text { Research and Treatment of Cancer Quality of Life } \\
\text { Questionnaire C30 [30]. } \\
\text { Module for men with prostate cancer - EORTC } \\
\text { QLQ-PR25 }\end{array}$ \\
\hline & Fatigue & $\begin{array}{l}\text { No fatigue, mild fatigue, moderate } \\
\text { fatigue, severe fatigue }\end{array}$ & PFS - Fatigue Scale of Piper [31]. \\
\hline & Self-esteem & $\begin{array}{l}\text { Satisfactory or high, medium, low or } \\
\text { unsatisfactory }\end{array}$ & Self-Esteem Scale for people with cancer [32]. \\
\hline & Sexual satisfaction & $\begin{array}{l}\text { Good sex life quality } \\
\text { Bad sex life quality }\end{array}$ & $\begin{array}{l}\text { IIFE } \\
\text { The International Index of Erectile Function [33]. }\end{array}$ \\
\hline & Depression & $\begin{array}{l}\text { No or minimal depression, mild } \\
\text { depression, moderate depression, } \\
\text { severe depression }\end{array}$ & BDI - Beck depression inventory [34]. \\
\hline & Descriptive variables & $\begin{array}{l}\text { Age, marital status, type of } \\
\text { treatment, education, income, } \\
\text { profession after diagnosis }\end{array}$ & Quiz created by the authors \\
\hline
\end{tabular}

will be observed. Outcomes that do not present a normal distribution will be dichotomized in compliance with the theoretical reference or through central trend distribution measures if it is lacking. We will then perform a univariate logistical regression between groups to adjust for possible confounding variables. Variables with $p$ values $<0.20$ will be selected and adjusted in the multiple logistic regression models. Variables with $p$ values $<0.05$ and/or clinical significance will be maintained in the model. 


\section{Discussion}

The incidence of prostate cancer has been increasing since the 1960s and it has become a growing public health concern. According to the National Cancer Institute [1], we are more aware of this disease because of the remarkable advances in its diagnosis. As a result, there is a need to develop strategies assisting patients undergoing treatment and after treatment. Although physical activity has been shown to positively impact disease symptoms and treatment side effects [13$15,19]$, most cancer survivors are not physically active. Programs focusing on physical activity are needed to assist cancer survivors in adopting and maintaining a new life style. However, exercise programs are limited in this patient population. This is compounded by limited infrastructure and resources, lack of patient information and patient understanding of the benefits of physical activity, and a lack of recommendations to men with prostate cancer to perform physical activity [24].

These factors make it difficult to implement interventions in this patient population. Research is warranted to identify the possibilities and benefits from physical activity intervention programs in reducing disease- and treatment-related side effects in patients with cancer. To our knowledge, no study has been conducted evaluating functional training interventions to improve the quality of life of men with prostate cancer. In addition to decreasing fatigue and depression-related symptoms, physical activity improves self-esteem, sexual satisfaction, and fitness. Previous studies have evaluated walking protocols [12] and aerobic exercises combined with resistance exercises [23] in men with cancer. Other studies have evaluated interventions that were not validated, including walking [19], aerobic training [14,17], and pelvic floor exercise [19], as well as support groups to improve quality-of-life $[13,16]$. These studies evaluating functional training protocols have reported improvements in quality-of-life, life style, abdominal circumference, and general fatigue, as well as a decrease in arterial pressure and anxiety.

This non-randomized functional training protocol for men with prostate cancer was developed to encourage physical activity and decrease side effects associated with prostate cancer treatment. It includes challenging exercises, but in a safe and progressive way that combines aerobic and resistance exercise based on previous research [11]. We expect that the intervention will improve the study participants' quality-of-life, fatigue, strength, balance, coordination, body mass control, physical fitness self-esteem, and depression-related symptoms.

With the significant increase of survival in men with prostate cancer, new knowledge is needed on the impact of physical activity. An increase of the quality of life and other related factors in men with prostate cancer may lead to new public policies motivating them to be more physically active after their diagnosis. Research may also add a new perspective to the current body of evidence on this subject and contribute to an appropriate physical activity protocol for this population.

\section{Ethical Statement}

View Ethical Statement.

\section{References}

1. Cancer national institute José Alencar Gomes Da Silva (INCA) (2015) CoordenaçãoDe Prevenção E Vigilância, Estimativa 2015, Incidência De Câncer No Brasil.

2. Orsini N, Bellocco R, Bottai M, Pagano M, Andersson SO, et al. (2009) A prospective study of lifetime physical activity and prostate cancer incidence and mortality. Br J Cancer 101: 1932-1938.

3. Magbanua MJ, Richman EL, Sosa EV, Jones LW, Simko J, et al. (2014) Physical activity and prostate gene expression in men with low-risk prostate cancer. Cancer Causes Control 25: 515-523.

4. Potosky AL, Legler J, Albertsen PC, Stanford JL, Gilliland FD, et al. (2000) Health outcomes after prostatectomy or radiotherapy for prostate cancer: results from the prostate cancer outcomes study. J Natl Cancer Inst 92: 1582-1592.

5. Potosky AL, Davis WW, Hoffman RM, Stanford JL, Stephenson RA, et al. (2004) Five-year outcomes after prostatectomy or radiotherapy for prostate cancer: the prostate cancer outcomes study. J Natl Cancer Inst 96: 1358-1367.

6. Langston B, Armes J, Levy A, Tidey E, Ream E (2013) The prevalence and severity of fatigue in men with prostate cancer: a systematic review of the literature. Support Care Cancer 21: 1761-1771.

7. Soares WTE (2011) Parameters, considerations and modulation of physical exercise programs for cancer patients - a systematic review. Rev Bras Med Esporte 17: 284-289.

8. World Health Organization (2011) Global recommendations on physical activity for health. 18-64 years old.

9. Guimarães GV, Ciolac EG (2014) Physical activity: practice this idea. Am J Cardiovasc Dis 4: 31-33.

10. Schmitz KH, Courneya KS, Matthews C, Demark-Wahnefried W, Galvão DA, et al. (2010) American College of Sports Medicine roundtable on exercise guidelines for cancer survivors. Med Sci Sports Exerc 42: 1409-1426.

11. Galvão DA, Spry N, Denham J, Taaffe DR, Cormie P (2014) Multicenter year-long randomized controlled trial of exercise training targeting physical functioning in men with prostate cancer previously treated with androgen suppression and radiation from Trog 03.04 Radar. Eur Urol 65: 856-864.

12. Hackshaw-Mcgeagh L, Lane JA, Persad R, Gillatt D, Holly JM, et al. (2016) Prostate cancer - evidence of exercise and nutrition trial (prevent): study protocol for a randomised controlled feasibility trial. Trials 17: 123.

13. Taylor CL, Demoor C, Smith MA, Dunn AL, Basen-Engquist $\mathrm{K}$, et al. (2006) Active for life after cancer: a randomized trial examining a lifestyle physical activity program for prostate cancer patients. Psychooncology 15: 847-862.

14. Reed SN, Robinson JW, Lau H, Stephenson L, Keats M, et al. (2010) Physical activity for men receiving androgen deprivation therapy for prostate cancer: benefits from a 16week intervention. Support Care Cancer 18: 591-599.

15. Herbert JR, Hurley TG, Harmon BE, Heiney S, Hebert CJ, 
et al. (2012) A diet, physical activity, and stress reduction intervention in men with rising prostate-specific antigen after treatment for prostate cancer. Cancer Epidemiol Prev 36: 128-136.

16. Taylor CL, Smith MA, de Moor C, Dunn AL, Pettaway C, et al. (2004) Quality of life intervention for prostate cancer patients: design and baseline characteristics of the active for life after cancer trial. Control Clin Trials 25: 265-285.

17. Gerset GM, Fossa SD, Dahl AA, Loge JH, Ensby T, et al. (2011) Effects of a 1-week inpatient course including information, physical activity, and group sessions for prostate cancer patients. J Cancer Educ 26: 754-760.

18. Mcgowan EL, North S, Courneya KS (2013) Randomized controlled trial of a behavior change intervention to increase physical activity and quality of life in prostate cancer survivors. Ann Behav Med 46: 382-393.

19. Mungovan SF, Huijbers BP, Hirschhorn AD, Patel MI (2013) Relationships between perioperative physical activity and urinary incontinence after radical prostatectomy: an observational study. BMC Urol 13: 67.

20. Campos MA, Neto BC (2004) Treinamento functional resistido: paramelhoria da capacidade funcional e reabilitação de lesões musculoesqueléticas. Rio de Janeiro, Revinter.

21. Naccarato A, Perchon L (2004) Estudo Do Impacto Da Disfunção Erétil Na Qualidade De Vida De Pacientes Pós Prostatectomia Radical. In: Convenção Brasil Latino América, Congresso Brasileiro e Encontro Paranaense De Psicoterapias Corporais. Anais.Cd-Rom.

22. Faul F, Erdfelder E, Lang AG, Buchner A (2007) G*Power 3: a flexible statistical power analysis program for the social, behavioral, and biomedical sciences. Behav Res Methods 39: 175-191.

23. Schega L, Torpel A, Hein N, Napiontek A, Wenzel C, et al. (2015) Evaluation of a supervised multi-modal physical exercise program for prostate cancer survivors in the rehabilitation phase: rationale and study protocol of the prolife study. Contemp Clin Trials 45: 311-319.

24. Baumann FT, Zopf EM, Bloch W (2012) Clinical exercise interventions in prostate cancer patients-a systematic review of randomized controlled trials. Support Care Cancer 20: 221-233.

25. Wells KF, Dillon EK (1952) The sit and reach - a test of back and leg flexibity. Res Quart 23: 115-118.

26. Pollock ML, Wilmore JH (1993) Exercícios na Saúde e na Doença: Avaliação e Prescrição para Prevenção e Reabilitação. MEDSI Editora Médica e Científica Ltda 233-362.

27. Osness WH, Adrian M, Clark B, Hoeger W, Raab D, et al. (1990) Functional Fitness Assessment for Adults Over 60 Years. The American Alliance for Health, Physical Education, Recreation and Dance (AAHPERD) Association for Research, Administration, Professional Councils, and Societies. Council on Aging and Adult Development.

28. Johnson BL, Nelson JK (1979) Practical measurements for evaluation in physical education. Minnesota: Burgess Publishing Company.

29. Pardini R, Matsudo S, Matsudo TAV, Andrade E, Braggion $\mathrm{G}$, et al. (1997) Validation of the international physical activity questionnaire (IPAQ): pilot study in Brazilian young adults. Med Sci Sports Exerc 29: 5-9.

30. Aaronson NK, Ahmedzai S, Bergman B, Bullinger M, Cull A, et al. (1993) The European Organisation for Research and Treatment of Cancer QLQ-C30: A quality-of-life instrument for use in international clinical trials in oncology. J Natl Cancer Inst 85: 365-376.

31. Mota DD, Pimenta CA, Piper BF (2009) Fatigue in Brazilian cancer patients, caregivers, and nursing students: a psychometric validation study of the Piper Fatigue Scale-Revised. Support Care Cancer 17: 645-652.

32. Curbow B, Somerfield M (1991) Use of the rosenberg self-esteem scale with adult cancer patients. Journal of Psychosocial Oncology 9: 113-131.

33. Rosen RC, Riley A, Wagner G, Osterloh IH, Kirkpatrick J, et al. (1997) The international index of erectile function (IIEF): a multidimensional scale for assessment of erectile dysfunction. Urology 49: 822-830.

34. beck At, Ward Ch, Mendelson M, Mock J, Erbaugh J (1961) An inventory for measuring depression. Arch Gen Psychiatry 4: 561-571. 